\title{
Recent advances in the management of peritoneal dialysis patients
}

\author{
Anjali B. Saxena, MD
}

Addresses: Department of Medicine, Division of Nephrology, Santa Clara Valley Medical Center, 751 S. Bascom Ave, San Jose, CA 95128, USA; Department of Medicine, Division of Nephrology, Stanford University, Stanford, CA, 94304, USA

Email: anjali.saxena@hhs.sccgov.org

Fl000Prime Reports 2015, 7:57 (doi:10.12703/P7-57)

All FI000Prime Reports articles are distributed under the terms of the Creative Commons Attribution-Non Commercial License (http://creativecommons.org/licenses/by-nc/3.0/legalcode), which permits non-commercial use, distribution, and reproduction in any medium, provided the original work is properly cited.

The electronic version of this article is the complete one and can be found at: http://f1000.com/prime/reports/m/7/57

\begin{abstract}
Peritoneal dialysis is a form of kidney dialysis that is used to remove accumulated metabolic waste products and water in patients with end stage kidney disease. Long-term exposure to high concentrations of glucose and its by-products, both found in peritoneal dialysis fluid, has been implicated in contributing to peritoneal damage over time, in turn limiting long-term use of the technique. Newer peritoneal dialysis solutions have been developed in the hope of reducing the unfavorable effects of peritoneal dialysis solutions. In vitro and in vivo studies have suggested that newer peritoneal dialysis fluids have salutary effects on the peritoneal membrane. Short-term clinical studies have also found some metabolic benefits of glucose-sparing regimens in chronic peritoneal dialysis. Mixed results have been found in studies examining whether newer peritoneal dialysis fluids reduce peritonitis rates. Long-term studies are needed to investigate whether newer peritoneal dialysis fluids provide better peritoneal dialysis technique and/or patient survival, compared to standard glucose-based peritoneal dialysis fluids.
\end{abstract}

\section{Introduction}

Peritoneal dialysis is a form of kidney dialysis that is used worldwide to treat patients with end stage renal disease (ESRD). Peritoneal dialysis prevalence varies regionally and, in developed areas, the proportion of dialysis patients on peritoneal dialysis generally ranges between 17 and $36 \%$, although it is as high as $79 \%$ in Hong Kong [1]. Peritoneal dialysis removes metabolic waste products and excess water in patients whose kidneys can no longer do so effectively, due to kidney failure. Excess water that typically accumulates in ESRD patients is removed in peritoneal dialysis by way of ultrafiltration; water transport into the peritoneal cavity is mostly driven through an osmotic gradient artificially generated by additives to the peritoneal dialysis solution, such as glucose. Glucose is readily available, inexpensive, and effective in promoting ultrafiltration of water in ESRD patients. Decades of use have demonstrated its general efficacy and safety; however, concerns about the limitations and long-term effects of peritoneal dialysis fluid (PDF) glucose, and other factors of the PDF that render it bio-incompatible, have stimulated research and development of alternate dialysis regimens to peritoneal dialysis.

\section{Standard peritoneal dialysis fluids}

The peritoneum is a semipermeable membrane that is composed of a thin layer of connective tissue overlain by a monolayer of mesothelial cells. The peritoneum covers the interior abdominal wall and intra-abdominal organs and has a surface area that approximates body surface area [2]. Peritoneal capillaries within the peritoneal membrane interstitium are in relatively close proximity to the mesothelial cell layer, and thus are close to the peritoneal cavity. Instillation of PDF into the peritoneal cavity creates solute concentration gradients that lead to the diffusion of highly concentrated "uremic" solutes from the blood supply into the PDF. After time, the PDF becomes saturated with solute and it can be discarded (drained out through the peritoneal catheter), followed by the instillation of fresh dialysate to begin the process anew. 
Standard PDF contains sterile water, electrolytes, lactate (converted to bicarbonate in vivo) added as a buffer to treat uremic acidosis, and glucose (osmotic agent). Intraperitoneal glucose is well tolerated by the patient and it is readily metabolized, thus preventing accumulation of toxic by-products. Glucose has been used as an osmotic agent in chronic peritoneal dialysis for more than 5 decades. Nevertheless, data illuminating the longterm local and systemic effects of glucose-containing PDF continue to accumulate, thus generating concern regarding the metabolic effects of glucose-PDF when used for long periods of time. Additionally, the efficacy of peritoneal dialysis, in terms of maintaining sustained ultrafiltration, and the health of the peritoneal membrane itself is thought to be in jeopardy with the longterm use of glucose-PDF.

With regards to the effect of glucose-PDF on the peritoneal dialysis system, the major difficulties are twofold; first, the high concentrations of glucose in PDF are directly cytotoxic to peritoneal mesothelial cells and cause diabetiform changes in the post-capillary venules [3]. The second problem with glucose-PDF involves the effects of standard heat sterilization of PDF during production; this autoclaving process generates numerous glucose degradation products (GDPs) in the PDF. Additional storage of PDF before use also allows generation of more GDPs [4-6]. GDPs are damaging because they are locally cytotoxic and also glycate local proteins to create advanced glycation end-products (AGEs). AGEs are known to be damaging both to the peritoneum and to the body after they enter the systemic circulation from the peritoneal cavity via passive diffusion. A list of GDPs commonly found in the peritoneal fluid of peritoneal dialysis patients can be found in Table 1. GDPs and AGEs in PDF are linked to damaging effects on the peritoneal membrane, including mesothelial cell loss, submesothelial fibrosis, calcification, vasculopathy, and diabetiform neoangiogenesis (implicated as one of the causes of ultrafiltration failure in peritoneal dialysis patients) [7]. All of these effects cumulatively decrease the effectiveness of the peritoneum

Table I. Some glucose-degradation products (GDPs) found in peritoneal dialysis fluid (PDF)

\section{GDP found in PDF}

Acetaldehyde

Formaldehyde

2-furaldehyde

Glyoxal

5-hydroxymethylfuraldehyde

Methylglyoxal

Valeraldehyde

3-deoxyglucosone

3,4-dideoxyglucosone-3-ene as a dialysis membrane, ultimately leading to transfer from peritoneal dialysis to hemodialysis. Furthermore, peritoneal GDPs and AGEs entering the circulation are thought to have systemic inflammatory and pro-oxidative effects, and may contribute to advanced atherosclerosis and accelerated loss of residual renal function [8-10] (Figure 1). It should be pointed out that, compared with hemodialysis patients, peritoneal dialysis patients typically retain residual renal function longer, often for months to years after the initiation of dialysis [11,12]. As little as $0.5 \mathrm{~mL} / \mathrm{min}$ of residual glomerular filtration has been associated with a $12 \%$ reduction in the relative risk of death [13].

The metabolic consequences of glucose-PDF include weight gain, lipid abnormalities, insulin resistance and possibly worsened glycemic control [14]. The glucose content of PDF ranges from $1360 \mathrm{mg} / \mathrm{dL}$ to $3860 \mathrm{mg} / \mathrm{dL}$. For a typical patient, PDF glucose is systemically absorbed on the order of about 100-300 $\mathrm{g}$ of glucose per day and these calories contribute between 12 and $34 \%$ of the daily energy intake of peritoneal dialysis patients. In catabolic or uremic patients these may be welcome calories but other patients, who have uncontrolled diabetes, insulin resistance, or obesity, may not benefit from this excess glucose [15].

\section{Alternative peritoneal dialysis fluids}

Numerous agents have been investigated as potential alternatives to replace glucose as the osmotic agent in PDF (e.g. sorbitol, xylitol, mannitol, dextran) but none have matched the efficacy and relative safety profile of glucose [15]. Nevertheless, the bio-incompatibility of standard glucose-PDF has led to continued efforts to develop alternative PDFs that either reduce the level of GDPs in dialysate to very low levels, or eliminate glucose entirely, thus preventing GDP formation. PDFs containing amino acid or icodextrin fluid in lieu of glucose are able to be GDP-free, while multi-chambered PDF bags have allowed the production of glucose-PDF with very low levels of GDP. Low GDP solution production makes use of the fact that GDP production during heat sterilization is less robust in acidic environments; manufacturers thus add a small amount of acid to the glucose PDF prior to autoclaving [16]. Unfortunately, the resultant acidic solution cannot be used directly in the peritoneum because the acidity causes intense pain upon instillation. Newer technology has allowed the development of multi-compartment PDF bags where in the glucose component can be heated and stored in a highly acidic environment ( $\mathrm{pH}$ 2.6-3) while the remainder of the PDFs (containing electrolytes and buffer) are in a separate compartment at a higher $\mathrm{pH}$; the two compartments are mixed just prior to use and, in doing so, achieve a more physiologic $\mathrm{pH}$ and excellent patient 


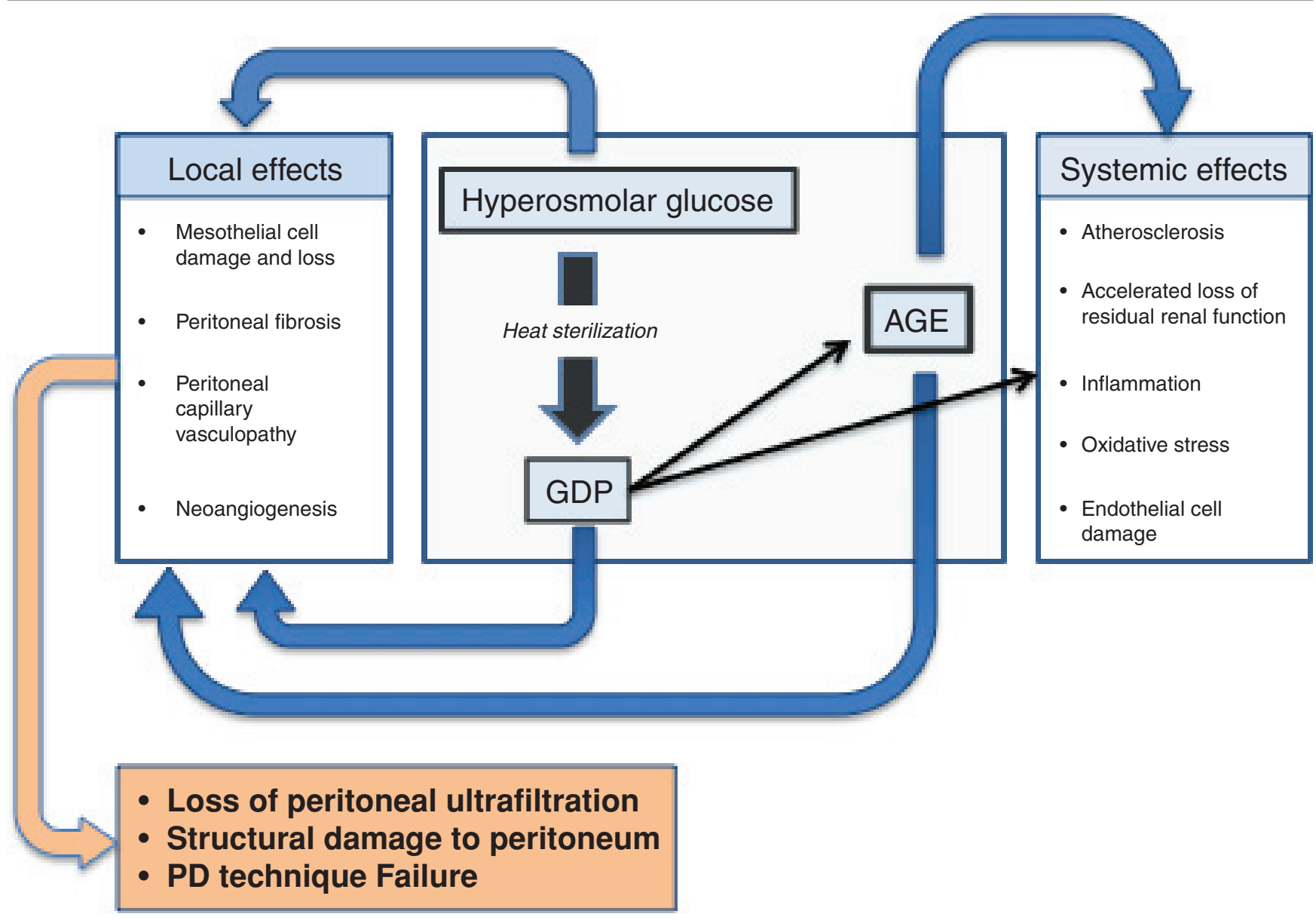

AGE, advanced glycosylation end product; GDP, glucose degradation product; PD, peritoneal dialysis; PDF, peritoneal dialysis fluid.

tolerability intraperitoneally. Multi-compartment PDFs are termed "biocompatible" PDFs since they contain fewer GDPs than standard PDFs; for example, they can achieve 3-deoxyglucosone concentrations less than $66 \mu \mathrm{mol} / \mathrm{L}$ compared to standard PDF, which contains $172-525 \mu \mathrm{mol} / \mathrm{L}[17]$.

Amino acid solution is a PDF that eliminates GDP entirely by substituting amino acid in lieu of glucose as the osmotic agent. Earlier formulations of amino acid solution were associated with marked metabolic acidosis and adverse nutritional effects; subsequent iterations of amino acid solution, with a higher concentration of buffer, have generally been better tolerated but are still associated with acidosis and are poorly tolerated when used more than once daily [18]. Icodextrin is another osmotic agent used in PDF to replace glucose; icodextrin is a starch-derived poly-glucose molecule that induces water transport into the peritoneal cavity via colloid forces. Icodextrin has a high peritoneal membrane reflection coefficient, and therefore remains in situ for long periods of time, thus enabling sustained ultrafiltration. It slowly exits the peritoneal cavity via lymphatic channels (it is not readily absorbed by the peritoneum), and is subsequently metabolized intracellularly. Although its metabolism does lead to energy absorption (i.e. carbohydrate calories), the metabolic profile of patients using icodextrin appears to be superior to that achieved with glucose-only PDF [19].

Biocompatible PDFs have been evaluated extensively in various scenarios. A summary of data obtained from in vitro, in vivo, experimental and clinical studies with biocompatible PDFs is found in Table 2. In general, biocompatible fluids appear to better preserve the peritoneum, reduce the formation of local AGE, and attenuate the development of peritoneal vasculopathy compared with standard glucose-PDF [18]. Clinical studies on the subject have recently been evaluated in a Cochrane review; the authors concluded that data from 
the available studies (all of sub-optimal quality) show that neutral $\mathrm{pH}$, low GDP PDF increases urine output after 12 months of use [20]. Of note, decreased peritoneal ultrafiltration rates appear to accompany increased urine output in these studies, suggesting that increased urine output may result from a shift in the location of water transport (from peritoneum to kidney) rather than improved renal function per se [21]. A recent meta-analysis of studies examining the effect of neutral $\mathrm{pH}$, low GDP solution on residual renal function was unable to find a significant benefit [17]. There is no definitive benefit of newer solutions on peritonitis rates; an equal number of studies show no difference in rates with newer PDF as show a benefit with biocompatible PDF. Finally, there has been no good trial to date to show a survival benefit with the use of biocompatible or glucose-sparing PDF.

\section{Recent investigation of glucose-sparing peritoneal dialysis regimens: promise of metabolic benefit?}

A prospective crossover trial in 22 diabetic peritoneal dialysis patients substituted one glucose-PDF exchange per day with either one amino acid solution or one icodextrin exchange, for a period of 8 weeks. Serum triglyceride levels decreased with both amino acid solution and icodextrin compared with glucose-PDF, and free fatty acids decreased during the use of amino acid solution [22]. A larger trial performed in Mexico prospectively evaluated the effect of one icodextrin per day versus standard PDF in 59 diabetic peritoneal dialysis patients. Compared to baseline, the icodextrin group (but not the control group) had reduced insulin need, lower fasting glucose, lower serum triglyceride level, lower hemoglobin (Hgb)A1c, and fewer adverse events related to glucose control [19].

More recently, the IMPENDIA and EDEN trials evaluated the clinical effects of a glucose-sparing peritoneal dialysis regimen in a multi-national study involving 251 peritoneal dialysis patients who were randomized to a glucose-sparing regimen or control group (Table 3) [23]. Baseline mean HgbA1c was not statistically different between the two groups (7.6\% in control, $7.7 \%$ in intervention). The main finding was an improvement in the mean glycated Hgb in the intervention group but not in the control group, in the order of a $0.5 \%$ improvement in 6 months (HbA1c decreased from 7.7 to $7.2 \%$ during the 6 months of study treatment). Secondary positive findings included a significant reduction in very low density lipoprotein, serum triglyceride and apolipoprotein B levels, as well as a trend towards a greater decrease in visceral fat volume in the intervention group. Unfortunately, over the 6-month study time, there was an unexpected decrease in mean serum albumin in the intervention group $(-0.5 \mathrm{~g} / \mathrm{L})$ compared with an increased serum albumin in the control group (0.6 $\mathrm{g} / \mathrm{dL}$ ). There were also more reported deaths in the intervention group than control (11 versus 5), although these were all considered unrelated to the study as judged by a study panel. Overall there was a significantly higher incidence of treatment-related adverse events, serious adverse events, and hypoglycemia, and more episodes of volume overload in the intervention group, plus a higher number of patients in the intervention group withdrew from the study.

It is not easy to organize a large multi-center trial in peritoneal dialysis, and it is disappointing that such an effort found a glucose-sparing peritoneal dialysis regimen to have more adverse events (particularly fluid overload) than a traditional glucose-only peritoneal dialysis regimen, despite metabolic benefits in the former. Nevertheless, we must consider that the trial was not performed in a tightly controlled experimental environment, but rather in many centers spanning 11 different countries, where local physician practice dictated volume management, and where these practices were by no means standardized. Fewer adverse events might well be seen with glucose-sparing regimens if close monitoring of fluid

Table 2. Selected findings from in vitro and in vivo studies of three different biocompatible peritoneal dialysis fluids (PDFs), categorized as beneficial (positive) or potentially harmful (negative)

\begin{tabular}{lll}
\hline & positive & negative \\
\hline pH-neutral, low GDP & Decreased mesothelial cell damage & Decreased peritoneal ultrafiltration \\
& $\begin{array}{l}\text { Decreased IL-6 in dialysate } \\
\text { Decreased circulating AGE } \\
\text { Improved anabolism and nutritional parameters (when combined with glucose) }\end{array}$ & $\begin{array}{l}\text { Increased IL-6 in dialysate } \\
\text { Increase serum urea } \\
\text { amino acid }\end{array}$ \\
& Preservation of mesothelial cell function & Metabolic acidosis \\
& & Inefficient ultrafiltration \\
Increased IL-6 in dialysate & Increased serum CRP \\
icodextrin & Preserved mesothelial cell morphology & \\
& Decreased fluid overload &
\end{tabular}

AGE, advanced glycosylation end product; CRP, C-reactive protein; GDP, glucose degradation product; interleukin, IL. 
Table 3. Overview of the IMPENDIA and EDEN trial study design [23]

\begin{tabular}{ll}
\hline IMPENDIA & EDEN \\
\hline Multi-site in I0 countries & Multi-site in Colombia only \\
$\mathrm{n}=$ I80 & $\mathrm{n}=7 \mathrm{I}$ \\
Study regimen: & Study regimen: \\
- I icodextrin bag & $\bullet$ I icodextrin bag \\
$\bullet$ I amino acid bag & $\bullet$ I amino acid bag \\
- 2 bicarbonate-based & -2 lactate-based \\
dextrose bags & dextrose bags \\
Controls: & Controls: \\
4 lactate-dextrose bags & 4 lactate-dextrose bags \\
\hline
\end{tabular}

status is upheld, and prompt changes are made to the peritoneal dialysis regimen when needed. Perhaps simply substituting a glucose bag for a non-glucose bag in a 1:1 fashion is not the answer; glucose-sparing regimens likely require additional adjustments to sustain adequate daily ultrafiltration. As an example, several studies have shown less peritoneal ultrafiltration with biocompatible PDF than standard glucose-PDF; when one biocompatible exchange is substituted for one glucose-PDF, the patient may require higher osmolality in one or all of the remaining standard-PDFs to reach daily ultrafiltration targets [24].

\section{Future directions and questions}

Many more questions than answers exist at this time regarding the role of biocompatible PDF and glucosesparing regimens in chronic peritoneal dialysis. Many of these questions will require longer term and larger studies to provide answers. Some suggestions for future investigation are provided below:

(a) Does glucose sparing improve the long-term use of the peritoneal dialysis technique?

(b) Does glucose sparing in non-diabetics who start peritoneal dialysis improve outcomes?

(c) How strictly should blood sugar be controlled in peritoneal dialysis patients, and do blood sugar targets vary for different subgroups of peritoneal dialysis patients?

(d) In diabetic peritoneal dialysis patients, what is the effect of glucose sparing on microvascular complications of diabetes (e.g. retinopathy, non-traumatic amputation)?

(e) In diabetic peritoneal dialysis patients, what is the long-term effect of glucose sparing on macrovascular complications of diabetes, including atherosclerotic cardiovascular disease?

\section{Abbreviations}

AGE, advanced glycosylation end product; ESRD, end stage renal disease; GDP, glucose degradation product; $\mathrm{Hgb}$, hemoglobin; PDF, peritoneal dialysis fluid.

\section{Disclosure}

Dr. Anjali B. Saxena has served on a speaker panel for Baxter Corporation.

\section{References}

I. Jain AK, Blake P, Cordy P, Garg AX: Global trends in rates of peritoneal dialysis. J Am Soc Nephrol 20I2, 23:533-44.

\section{FlOOOPrime}

RECOMMENDED

2. Nolph and gokal's textbook of peritoneal dialysis. 3rd Edition. New York: Springer; 2008.

3. Gotloib L, Wajsbrot V, Shostak A, Kushnier R: Morphology of the peritoneum: effect of peritoneal dialysis. Perit Dial Int 1995, I5: S9-II; discussion S-2.

4. Linden T, Cohen A, Deppisch R, Kjellstrand P, Wieslander A: 3,4Dideoxyglucosone-3-ene (3,4-DGE): a cytotoxic glucose degradation product in fluids for peritoneal dialysis. Kidney Int 2002, 62:697-703.

\section{FlOOOPrime}

\section{RECOMMENDED}

5. Nilsson-Thorell $\mathrm{CB}$, Muscalu $\mathrm{N}$, Andren AH, Kjellstrand PT, Wieslander AP: Heat sterilization of fluids for peritoneal dialysis gives rise to aldehydes. Perit Dial Int 1993, 13:208-13.

6. Erixon $M$, Linden $T$, Kjellstrand $P$, Carlsson $O$, Ernebrant $M$, Forsback G, Wieslander A, Jonsson JA: PD fluids contain high concentrations of cytotoxic GDPs directly after sterilization. Perit Dial Int 2004, 24:392-8.

\section{FlOOOPrime}

\section{RECOMMENDED}

7. Krediet RT, Zweers MM, van der Wal AC, Struijk DG: Neoangiogenesis in the peritoneal membrane. Perit Dial Int 2000, 20(Suppl 2): SI $9-25$.

\section{FlOOOPrime}

\section{RECOMMENDED}

8. Fusshoeller A: Histomorphological and functional changes of the peritoneal membrane during long-term peritoneal dialysis. Pediatr Nephrol 2008, 23:19-25.

\section{FlOOOPrime}

\section{RECOMMENDED}

9. Breborowicz A, Pawlaczyk K, Polubinska A, Gorna K, Wieslander A, Carlsson O, Tam P, Wu G: Effect of peritoneal dialysis on renal morphology and function. Nephrol Dial Transplant 2006, 21:3539-44.

\section{FIOOOPrime}

\section{RECOMMENDED}

10. Justo $P$, Sanz $A B$, Egido J, Ortiz A: 3,4-Dideoxyglucosone-3-ene induces apoptosis in renal tubular epithelial cells. Diabetes 2005, 54:2424-9.

\section{FlOOOPrime}

\section{RECOMMENDED}

1I. Jansen MA, Hart AA, Korevaar JC, Dekker FW, Boeschoten EW Krediet RT, Group NS: Predictors of the rate of decline of residual renal function in incident dialysis patients. Kidney Int 2002, 62: 1046-53.

FlOOOPrime RECOMMENDED

12. Rottembourg J, Issad B, Gallego JL, Degoulet P, Aime F, Gueffaf B, Legrain $M$ : Evolution of residual renal function in patients 
undergoing maintenance haemodialysis or continuous ambulatory peritoneal dialysis. Proc Eur Dial Transplant Assoc 1983, 19:397-403.

13. Bargman JM, Thorpe KE, Churchill DN: Relative contribution of residual renal function and peritoneal clearance to adequacy of dialysis: a reanalysis of the CANUSA study. J Am Soc Nephrol, 12:2158-62

\section{FlOOOPrime}

\section{RECOMMENDED}

14. Skubala A, Zywiec J, Zelobowska K, Gumprecht J, Grzeszczak W: Continuous glucose monitoring system in 72-hour glucose profile assessment in patients with end-stage renal disease on maintenance continuous ambulatory peritoneal dialysis. Med Sci Monit 2010, 16:CR75-83.

\section{FlOOOPrime}

15. Holmes C], Shockley TR: Strategies to reduce glucose exposure in peritoneal dialysis patients. Perit Dial Int 2000, 20(Suppl 2): S37-4I.

16. Kjellstrand P, Martinson E, Wieslander A, Kjellstrand K, Jeppsson E, Svensson E, Jarkelid L, Linden T, Olsson LF: Degradation in peritoneal dialysis fluids may be avoided by using low $\mathrm{pH}$ and high glucose concentration. Perit Dial Int 200I, 21:338-44.

\section{FlOOOPrime}

17. Cho Y, Badve SV, Hawley CM, Wiggins K, Johnson DW: Biocompatible peritoneal dialysis fluids: clinical outcomes. International journal of nephrology 2012, 2012:812609.

18. Chaudhary K, Khanna R: Biocompatible peritoneal dialysis solutions: do we have one? Clin J Am Soc Nephrol 2010, 5:723-32.

\section{FlOOOPrime}

19. Paniagua $R$, Ventura MD, Avila-Diaz M, Cisneros A, VicenteMartinez M, Furlong MD, Garcia-Gonzalez Z, Villanueva D, Orihuela O, Prado-Uribe MD, Alcantara G, Amato D: Icodextrin improves metabolic and fluid management in high and highaverage transport diabetic patients. Perit Dial Int 2009, 29:422-32.

\section{FlOOOPrime}

\section{RECOMMENDED}

20. Cho Y, Johnson DW, Craig JC, Strippoli GF, Badve SV, Wiggins KJ: Biocompatible dialysis fluids for peritoneal dialysis. Cochrane Database Syst Rev 2014, 3:CD007554.

\section{FlOOOPrime
RECOMMENDED}

21. Bargman JM: Slouching towards Bethlehem: the beast of biocompatibility. Nephrol Dial Transplant 2010, 25:2050-I.

22. Martikainen T, Teppo AM, Gronhagen-Riska C, Ekstrand A: Benefit of glucose-free dialysis solutions on glucose and lipid metabolism in peritoneal dialysis patients. Blood Purif 2005, 23:303-10.

\section{FlOOOPrime}

RECOMMENDED

23. Li PK, Culleton BF, Ariza A, Do JY, Johnson DW, Sanabria M, Shockley TR, Story K, Vatazin A, Verrelli M, Yu AW, Bargman JM, Impendia, Groups ES: Randomized, controlled trial of glucosesparing peritoneal dialysis in diabetic patients. J Am Soc Nephrol 2013, 24:1889-900.

FlOOOPrime

RECOMMENDED

24. Blake PG, Jain AK, Yohanna S: Biocompatible peritoneal dialysis solutions: many questions but few answers. Kidney Int 2013, 84:864-6. 